\title{
Simulating Light Adaptation in the Retina with Rod-Cone Coupling
}

\author{
Kendi Muchungi and Matthew Casey \\ University of Surrey, Guildford, Surrey \\ GU2 7XH, United Kingdom. +44 (0)1483 300800 \\ $\{\mathrm{k}$.muchungi, m. casey\}@surrey.ac.uk \\ http://www.surrey.ac.uk/computing
}

\begin{abstract}
The retina performs various key operations on incoming images in order to facilitate higher-level visual processing. Since the retina outperforms existing image enhancing techniques, it follows that computational simulations with biological plausibility are best suited to inform their design and development, as well as help us better understand retina functionality. Recently, it has been determined that quality of vision is dependant on the interaction between rod and cone pathways, traditionally thought to be wholly autonomous. This interaction improves the signal-to-noise ratio (SNR) within the retina and in turn enhances boundary detection by cones. In this paper we therefore propose the first cone simulator that incorporates input from rods. Our results show that rod-cone convergence does improve SNR, therefore allowing for improved contrast sensitivity, and consequently visual perception.
\end{abstract}

Keywords: Photoreceptors, Cones, Rods, Rod-Cone Coupling

\section{Introduction}

Despite a century of effort, we still do not fully understand visual processing in the retina. Recently, it was determined that the communication between light sensitive neurons in the retina; rods and cones, which were traditionally thought to be autonomous, is important to the quality of vision [12. Here, signals from rods and cones integrate thereby enabling the retina to operate over a very large dynamic range of light intensities. However, the extent of the interaction between these two distinct pathways under varying light conditions is still unknown 8 .

The retina performs various key operations on incoming images in order to facilitate higher-level visual processing. One of the most important is light adaptation [11. Adaptation is the process by which photoreceptors adjust to ambient light levels that can range from 1 to $10^{8}$ factors of light intensity, and requires more than the iris adjusting pupil size. It requires photoreceptors to adapt the level of their sensitivity so as to keep responses to visual stimuli approximately the same regardless of the changing levels of illumination, allowing for higher-level processing to continue without overt influence from continuously changing light levels [4]. 


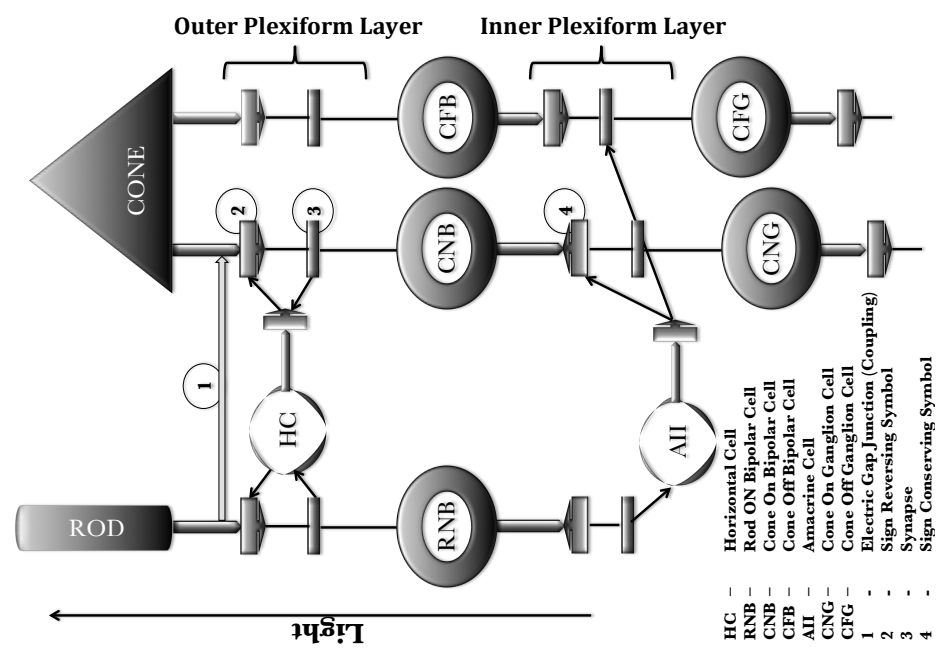

Fig. 1: A section through the human eye with a schematic of neurons in the retina outer layer

Light adaptation is achieved by the following processes: the conversion of light into electrical signals that the brain can understand in a process referred to as photo-transduction; transfer of visual information between photoreceptors due to their coupling; and data reduction, via negative feedback to the photoreceptors from Horizontal Cells (HCs). Coupling, more specifically rod-cone coupling, is important because it helps improve SNR. Specific to photoreceptors, a good SNR helps increase the dynamic range of visual perception [11.

Since the retina outperforms existing man made image acquisition devices [3], it follows that computational simulations with biological plausibility are best suited to inform the design and development of image enhancement techniques, as well as help us better infer retina functionality. Existing retinal models geared for biological precision have used established computational techniques to simulate light adaptation, contrast gain control and spike generation [10[13/16]14]. For example, Shah and Levine [10] modelled light adaptation by computing a local spatiotemporal ambient intensity $(A)$, with cone-cone coupling and $\mathrm{HC}$ output as a function of $A$ and then subtracting negative HC feedback using a Difference of Gaussians (DoG). This model was the first to simulate dynamic adaptation of the receptive field sizes as a function of the level of illumination (spatiotemporal contrast). Wilson [13] employed a cascade of low-pass filters, a linear negative feedback network and the Michaelis-Menten function. This is the only model that simulates ON and OFF parallel channels, an element that becomes relevant in simulating contrast gain control in the retina. Zaghloul and Boahen [16] present a physical implementation that uses nMOS transistors to enable replication of the photo-transduction process, adapt synaptic strengths for cone-cone coupling and incorporate subtractive $\mathrm{HC}$ feedback. Wohrer and Kornprobost [14] just like [10,13] use similar mathematical operations but in- 
corporate band-pass temporal filters to replicate light adaptation but do not simulate cone-cone coupling.

These models all simulate some or all of the aspects that contribute to light adaptation, albeit using different combinations of the same computations. However, they do not allow for the integration of rods and cones, whose inclusion would improve perception of scenes with a large range in luminance through contrast enhancement. In this paper we therefore propose a cone simulator that receives input from rods to understand the extent and varying degree this convergence contributes to improved visual perception. Section 2 explains how we model light adaptation. In section 3, we evaluate our simulator's performance by making comparisons with actual cone output $[9$ and with that of an existing model [10]. We then explore our contrast enhancement capability in comparison to a standard image processing technique. Finally, in section 4 we give a summary and proffer possible future work that emanate from our research.

\section{Method}

Our model comprises processes that continuously transform incoming signals. Participating processes consist successive spatiotemporal maps and correspond with photo-transduction, cone-cone coupling and negative HC feedback. The incoming light intensity takes the profile $I(x, y, t)$, defined for every spatial point $(x, y)$ of the photoreceptors at time $t$. Our simulator uses trolands (td) to quantify light intensity, with the spatial domain discretised into pixels where each pixel width is representative of a rod or a cone between $5^{\circ}$ to $10^{\circ}$ from the fovea.

\subsection{The Photo-Transduction Process}

The first stage in our simulator is the Photo-transduction process. Previous models [10[16]13 14 have modelled this process by using either a combination of the Michaelis-Menten function and/or a temporal low-pass filter thereby transforming the incoming signal into our Temporal Ambient Intensity, $T$ 2.1). The Michaelis-Menten function was used because it best replicates saturation in photoreceptors, whereas the temporal low-pass filter replicates how photoreceptors filter out unwanted information pertaining to the level of illumination. It was for these reasons we determined that the photo-transduction process would best be modelled by the aforementioned computations, with the exception that we modified the Michaelis-Menten function by incorporating the temporal low-pass filter $K$, because this gave a better fit with biology 8 .

$$
\begin{gathered}
T(x, y, t)=\left(\frac{I(x, y, t)^{n}}{I(x, y, t)^{n}+\left(\frac{k_{r} K\left(t ; \tau_{\text {cone } e}\right)}{k_{b}}+k_{r}\right)^{n}}\right) T_{\text {max }} \\
K\left(t ; \tau_{\text {cone }}\right)=\frac{e\left(\frac{-t}{\tau_{\text {cone }}}\right)}{\tau_{\text {cone }}}
\end{gathered}
$$


Our pigment bleach time constant $\left(\tau_{\text {cone }}\right)$ takes the value $1 \mu \mathrm{s}$, which is equivalent to cone integration time as used by [10, whereas the rod time constant is $4 \times \tau_{\text {cone }}$ consistent with biology [15. We then calculate our spatial ambient intensity, $S$, by convolving the input intensity with a low-pass temporal filter using rod pigment bleaching time constant, which is the main element of our contribution. $\sigma$ is the dynamic radius of the Gaussian, which replicates local adaptation to the dynamic range of intensities within the visual stimuli. $*$ denotes convolution.

$$
\begin{gathered}
S(x, y, t)=I(x, y, t) * \sigma_{s}(x, y, t) K\left(t ; \tau_{\text {Rod }}\right) \\
\sigma_{S}(x, y, t)=\alpha_{\text {rod }} I(x, y, t) \\
K\left(t ; \tau_{\text {rod }}\right)=\frac{e\left(\frac{-t}{\tau_{\text {rod }}}\right)}{\tau_{\text {rod }}}
\end{gathered}
$$

$\alpha_{\text {rod }}=100$ and is the weighting for the rod input our source of spatial ambient intensity, consistent with the electrical coupling between rods to cones. We use 100 because rods are about 100 times more sensitive to light than cones 2. Our final spatiotemporal ambient intensity, $A$, is then obtained by a weighted combination of $T$ and $S . A$ is used to adapt the sensitivity of photoreceptors during photo-transduction to match the local illumination levels. We use the following weightings: $\alpha_{T}=0.90$ and $\alpha_{S}=1-\alpha_{T}$.

$$
A(x, y, t)=\alpha_{T} T(x, y, t)+\alpha_{S} S(x, y, t)
$$

\subsection{Cone-Cone Coupling}

The extent of cone coupling is a function of 1) the local spatiotemporal ambient intensity, $A, 2)$ the distance from the fovea. This matches data on psychophysically measured visual acuity, so that we use the diffusion process employed by [10], which incorporates these elements and is shown by (2.7). To ensure cone coupling remains bounded for all illumination levels our data uses an exponent $n_{A}$, constants $Q_{o}$ and $\delta_{n_{A}}$ as has been used by Shah and Levine [10]. $\frac{3}{2}$ is used because it best describes the ratio of cones that influence a single cone [5]. The constants used are: $Q_{o}^{n_{A}}=65 \mathrm{td}, \delta_{n_{A}}=0.01$ and $n_{A}=0.50$.

$$
C_{\text {Coupling }}(x, y, t)=\frac{3}{2}\left(\frac{A(x, y, t)_{n_{A}}+Q_{o}^{n_{A}}}{A(x, y, t)_{n_{A}}+\delta^{n_{A}}}\right)
$$

\subsection{HC Feedback}

Before the final output can be calculated, we obtain cone output response without HC subtractive feedback $\left(C_{N o H C}\right)$, given by $(2.8)$. This response is obtained by convolving $A$ dynamically with the transformed $A$ due to cone-cone coupling and multiplying this by the temporal latency inherent in cones.

$$
C_{N o H C}(x, y, t)=A(x, y, t) * C_{\text {Coupling }}(x, y, t) K\left(t ; \tau_{\text {cone }}\right)
$$




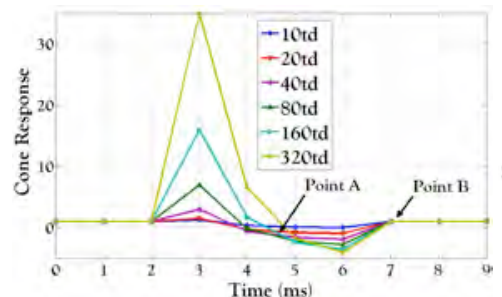

(a)

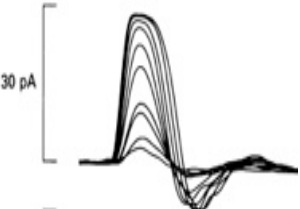

(b)

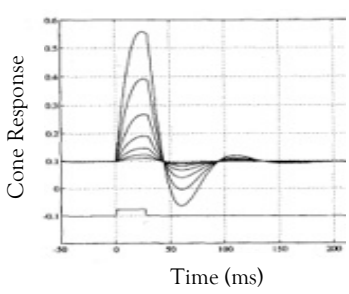

(c)

Fig. 2: (a) Cone output from our simulator in response to flash intensities 10/20/40/160/320td, (b) a Monkey's cone response as flash intensity were progressively doubled and (c) Shah-Levine model 9 cone response to $30 \mathrm{~ms}$ full-field flashes at flash intensities of $10 / 20 / 40 / 160 / 320 / 640$ td

Final cone response $\left(C_{\text {Response }}\right)$ is calculated by subtracting weighted HC output from $C_{N o H C}$ (essentially a DoG), whereas HC output is a weighting of $C_{N o H C}$.

$$
\begin{gathered}
C_{\text {Response }}(x, y, t)=C_{N o H C}(x, y, t)-\alpha_{H C} H_{\text {Response }}(x, y, t) \\
H_{\text {Response }}(x, y, t)=H(x, y, t) K\left(t ; \tau_{H C}\right)
\end{gathered}
$$

We can therefore calculate $C_{\text {Response }}$ by using (2.11), where $\alpha_{H C}$ is equal to the dynamically changing level of illumination and $\tau_{H C}$ is the HC time constant given by $20 \mathrm{~ms}$, as used by Shah and Levine [10.

$$
C_{\text {Response }}(x, y, t)=C_{N o H C}(x, y, t)\left(1-\alpha_{H C} K\left(t ; \tau_{H C}\right)\right)
$$

\section{Results}

To evaluate our simulator's viability, we used the Threshold Versus Intensity (tvi) function, which has been used by [910] to evaluate cone/simulator performance and as advocated by Hood 4 . We then compare the contrast enhancement performance of the simulator against a traditional computational technique.

\subsection{Biological Validation}

The tvi function is an important measure for field adaptation because it calculates the cone output in response to flash intensity [4. To a steady background, a flash is introduced to evaluate flux in sensitivity induced by changes in ambient light. To a background intensity of $0.0028 \mathrm{td}$, characteristic of mesopic luminance, we introduced flash intensities obtained by $10 \cdot 2^{p}$ where $p$ is an integer $(0.00 \leq p \leq 5.00)$. Figure 2 shows cone responses from our simulator compared to a monkey cone [11] and Shah-Levine's simulations [10].

For all three, there is a neural response corresponding to the perception of contrast during flash onset represented by the peaks. The subsequent decline in neural response is due to the termination of flash intensities. The further decline beyond the point of response to background intensity (undershoot) is neural response to lateral inhibition introduced by negative $\mathrm{HC}$ feedback. The points labelled A and B help draw attention to variations between the three graphs. 


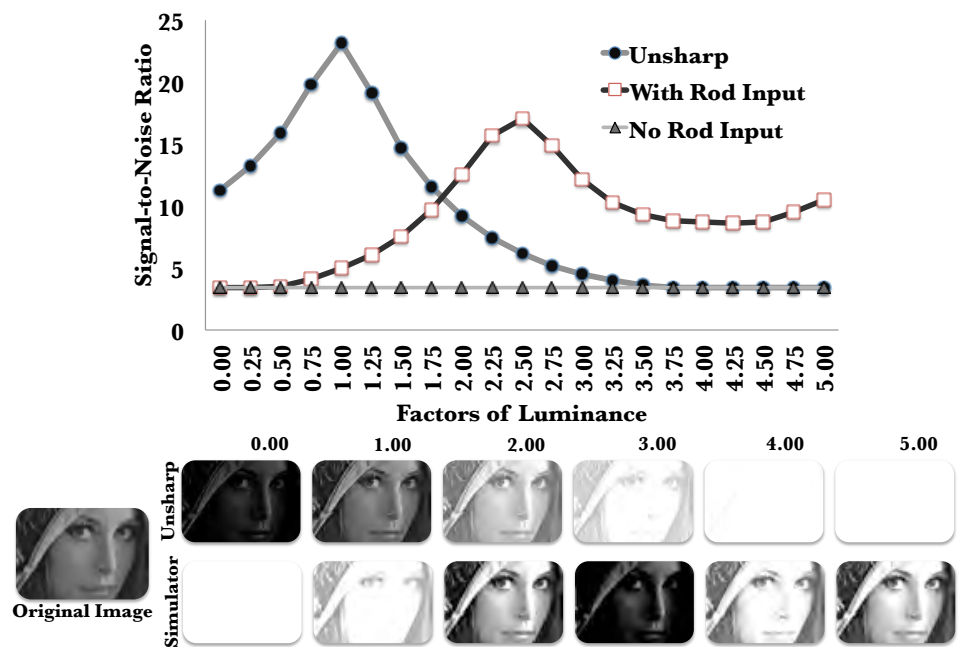

Fig. 3: Comparison of SNR for UM and our simulator (with and without rod input) and the results from processing the same brightness-adjusted image. Note that UM copes better with darker images and our simulator with brighter images.

We note that at Point $\mathrm{A}$ the response to each of the different flash intensities causes the cone response to drop below the background response at different times. This is the same as recorded in biology but was not achieved in Shah and Levine's simulation, where the crossing points are the same. Our simulator achieves this because it allows for different diffusion rates in response to the varying flash intensities. At Point B our simulator returns to the steady state at background intensity response. This is different to both biology and Shah and Levine's simulator and is representative of visual masking [6], whose modelling forms part of our future work. With regards to the tvi function we have shown that our simulator behaves in a manor similar to biology beyond the capability of Shah and Levine's model.

\subsection{Comparison with Image Contrast Enhancement}

The tvi function helps establish biological viability of our simulator, however, the question to muse over is, does our simulator offer any insights for image processing? To this end, we compare our simulator's performance against that of a standard contrast enhancement filter algorithm, the Unsharp mask (UM) [1. We adapted the brightness of a segment of the Lena image $(200 \leq x \leq$ $399,170 \leq y \leq 369$ ) by 1 ) getting the mean pixel intensity of the image, 2) subtracting the mean and 3) adding back a fraction of the mean. We chose factors from 0.00 to 5.00 in steps of 0.25 to give a full range of brightness but such that distinguishing features in the image were still visible. We processed the same brightness-adjusted image using UM and our simulator (with rod input and without) to obtain the SNR for each resulting image compared to the original input image. The SNR shows whether the contrast enhancement has resulted in an adjusted image which is closer to the original (a high value is better). 
From the results (Figure 3), we established that the minimum possible SNR is 3.42 for both UM (above brightness factor 4.75) and our simulator when no rod input is applied. The best contrast enhancement is obtained by the UM when the brightness factor is 1.00 (SNR 23.15) and hence the adjusted image is the same as the original. Conversely the simulator obtained its best performance (SNR 17.08) at brightness factor 2.50.

UM sees a steady decline in performance from factor 1.00 to level out at 4.00 (SNR 3.42), while the simulator's performance oscillates with a minimum at 4.25 (SNR 8.66), rising beyond this (to SNR 10.49). We can therefore see that our simulator copes much better with bright images, while the UM has a single peak centred on the original image brightness. While the simulator does not cope well with darker images compared to UM, it does demonstrate how contrast is extracted from bright images (Figure 3 ) and that this requires the rod input, which distinguishes our simulator from others.

\subsection{Discussion}

The experiments above show that our simulator has biological precision and offers better performance because we include rod input. They demonstrate that rod-cone coupling allows for a larger range of luminance to be dealt with more effectively [12. This performance translates to image processing when compared to UM when the level of luminance is high, because our simulator has been adapted for sensitivity to contrast especially when the level of luminance increases. This is due to rod input, which enhances light adaptation enabling perception of contrast at these high intensities. However, contrast is extracted at later timesteps demonstrating the tradeoff between instantaneous contrast enhancement (UM) and enhancement over time. Whereas during dark adaptation, rod-cone coupling is disabled and our dominant light sensitive neuron is the rod, an aspect we do not model and explains our poor performance during dark adaptation. We can also see in Figure 3 at high luminance SNR rises. This suggests that our technique can cope with a far higher range of luminance in a single image compared to image acquisition devices, which require different exposures for a single image to obtain a greater dynamic range [7].

\section{Conclusion}

In this paper we presented a cone simulator that receives rod and negative $\mathrm{HC}$ input. Input from the rod adjusts for spatial ambient intensity, and from the $\mathrm{HC}$ ensures data reduction. Both these inputs, in conjunction with pigment bleaching and cone-cone coupling, enable dynamic adaptation of the receptive fields with respect to the level of light intensity and help attain localised light adaptation by ensuring perceptual contrast constancy. To simulate light adaptation, we used a combination of low-pass temporal filters, a DoG, the Michaelis-Menten function and a diffusion process that measures visual acuity. To evaluate our simulator, we employed the tvi-function to ascertain biological viability and used 
UM to evaluate performance against existing contrast enhancing algorithms. We demonstrated the importance of rod-cone coupling in light adaptation and how this might be applied in image processing. The next stage is to incorporate neural models of bipolar, amacrine and ganglion cells so that output can fully simulate the functions of the retina, which are light adaptation, contrast gain control and spike generation.

\section{References}

1. Arici, T., Altunbasak, Y.: Image local contrast enhancement using adaptive nonlinear filters. In: Image Processing, 2006 IEEE International Conference on. pp. $2881-2884(2006)$

2. Baylor, D.: How photons start vision. Proceedings of the National Academy of Sciences 93(2), 560-565 (1996)

3. Graf, H.G., Dollberg, A., Spüntrup, J.D., Warkentin, K.: HDR Sub-retinal Implant for the Vision Impaired High-Dynamic-Range (HDR) Vision, Springer Series in Advanced Microelectronics, vol. 26, pp. 141-146. Springer Berlin Heidelberg (2007)

4. Hood, D.C.: Lower-level visual processing and models of light adaptation. Annual Review of Psychology 49, 503-535 (1998)

5. Hood, D., Birch, D.: Human cone receptor activity: The leading edge of the a-wave and models of receptor activity. Visual Neuroscience 10(05), 857-871 (1993)

6. Macknik, Stephen L.Livingstone, M.S.: Neuronal correlates of visibility and invisibility in the primate visual system. Nature Neuroscience 1(2), 144 (1998)

7. Mantiuk, R., Myszkowski, K., Seidel, H.P.: A perceptual framework for contrast processing of high dynamic range images. ACM Trans. Appl. Percept. 3(3), 286308 (Jul 2006), http://doi.acm.org/10.1145/1166087.1166095

8. Schiller, P.H.: Parallel information processing channels created in the retina. Proceedings of the National Academy of Sciences (2010)

9. Schnapf, J., Nunn, B., Baylor, D.: Visual transduction in cones of the monkey macaca fascicularis 427(1), 681-713 (1990)

10. Shah, S., Levine, M.D.: Visual information processing in primate cone pathways. i. a model. Systems, Man, and Cybernetics, Part B: Cybernetics, IEEE Transactions on 26(2), 259-274 (1996)

11. Shapley, R., Enroth-Cugell, C.: Visual adaptation and retinal gain controls, vol. 3, book chapter 9, pp. 263-346. Pergamon (1984)

12. Trümpler, J., Dedek, K., Schubert, T., de Sevilla Müller, L.P., Seeliger, M., Humphries, P., Biel, M., Weiler, R.: Rod and cone contributions to horizontal cell light responses in the mouse retina. Journal of Neuroscience 28(27), 6818-6825 (2008)

13. Wilson, H.R.: A neural model of foveal light adaptation and afterimage formation. Visual Neuroscience 14(03), 403-423 (1997)

14. Wohrer, A., Kornprobst, P.: Virtual retina: A biological retina model and simulator, with contrast gain control. Journal of Computational Neuroscience 26(2), 219-249-249 (2009)

15. Yau, K.: Phototransduction mechanism in retinal rods and cones: The friedenwald lecture. Investigative Ophthalmology and Visual Science 35(1), 9-32 (1994)

16. Zaghloul, K.A., Boahen, K.: A silicon retina that reproduces signals in the optic nerve. Journal of Neural Engineering 3(4), 257-67 (2006) 